\title{
Die papierchromatographische Bestimmung der einzelnen Säure- und Zuckerarten als Grundlage für die Auslese auf Wohlgeschmack bei Obst, Beerenobst und Gemüse*
}

\author{
Von Chr. Jordan, F. Korte, R. von SENGBusch
}

Mit 24 Textabbildungen

Die Züchter von Obst, Beerenobst und Gemüse sind seit langem daran interessiert, die Auslese auf geschmackliche Qualität mit Hilfe chemischer Methoden durchzuführen. Nur beim Vorhandensein chemischer Schnellbestimmungsmethoden läßt sich eine wirksame Auslese oder Vorauslese auf Geschmack an einem zahlenmäßig großen Material vornehmen.

Wir haben vor über 20 Jahren bereits einmal den Versuch gemacht, solche chemischen Methoden zur Bestimmung der Zucker- und Säurekomponenten des Geschmacks zu entwickeln.

Bei der Säure scheiterten diese Versuche daran, da $B$ die Schnellbestimmungsmethoden nur zur Bestimmung des Gesamtsäuregehaltes brauchbar waren. Beim Zucker ließen sich weder die Gesamtmenge noch die einzelnen Zuckerarten qualitativ, geschweige denn quantitativ bestimmen. Sowohl die FeHLING'sche als auch die polarimetrische und refraktometrische $\mathrm{Me}$ -

\footnotetext{
* Diese Arbeiten wurden mit Unterstützung der
} Deutschen Forschungsgemeinschaft durchgeführt. thode sind für die Bestimmung des Süßwertes ungeeignet.

Wir haben dann die organoleptische Methode der Geschmacksbestimmung im großen in der Züchtung angewendet und dafür zunächst die Menschen ausgelesen, die für eine solche Prüfung besonders gut geeignet waren; d. h. die, die

I. in der Lage waren, den Geschmack von Früchten reproduzierbar zu bestimmen,

2. nur geringe Ermüdungserscheinungen bei diesen Prüfungen aufwiesen, und

3. in der Lage waren, ohne körperliche Schäden eine große Zahl von Prüfungen durchzuführen.

Mit Hilfe der organoleptischen Methode haben wir jährlich etwa Ioooo Prüfungen von Erdbeerklonen durchgeführt. Wir haben Klone gesucht und gefunden, deren Früchte nach dem Gefrieren und Wiederauftauen nicht den typischen Gefriergeschmack besitzen.

Der Wunsch nach dem Vorhandensein geeigneter chemischer Schnellbestimmungsmethoden des Ge- 
schmacks blieb bestehen. Nur Schnellbestimmungsmethoden, die die einzelnen Zucker- und Säurearten sowohl qualitativ als auch quantitativ erfassen, wären für eine züchterische Vorauslese auf Wohlgeschmack zu gebrauchen.

Wir sind uns darüber im klaren, daß der Geschmack eine komplexe Eigenschaft ist. Der Säure- und Zuckergehalt stellen nur zwei Teilkomplexe dar, die ergänzt werden durch den Komplex der Aromastoffe, der Basen und Salze, über deren geschmackliche Auswirkung wir erst sehr wenig wissen, und durch die morphologischen Eigenschaften wie Konsistenz des Fruchtfleisches, Art der Fruchtschale usw.

Wir wollen uns in dieser Arbeit nur mit clem Säureund Zuckerkomplex beschäftigen.

Der Gesamtsäuregehalt ist kein Gradmesser für den sauren Geschmack. Wir wissen, daß die einzelnen Säurearten spezifische Geschmacksreaktionen auslösen, daß die unteren Schwellenwerte, bei denen sie wirksam werden, verschieden hoch liegen und daß bei gleicher Konzentration nicht bei jeder Säure die gleiche Säuregeschmacksempfindung ausgelöst wird.

Von den Zuckerarten wissen wir, daß auch bei diesen die Bestimmung des Gesamtzuckers nicht mit der Süße übereinstimmt. Die einzelnen Zuckerarten haben verschiedene Süßwerte. Die Süßwerte, die die einzelnen Autoren für die verschiedenen Zuckerarten festgestellt haben, stimmen nicht miteinander überein. Hieraus ist $z u$ schließen, daß es sehr schwer ist, objektiv die Süße eines jeden Zuckers organoleptisch zu bestimmen. Übereinstimmend wird aber festgestellt, daB die Zuckerarten mit ihrem Süßwert in folgender Reihenfolge abnehmen: Fructose, Saccharose, Glucose, Maltose, Lactose.

BECKER und HฐRzog geben die entsprechenden Süßwerte der 5 Zuckerarten wie folgt an: 173, roo, 74 , 32,16 .

Der Züchter möchte jede einzelne Zuckerart genau wie die Säuren quantitativ erfassen, um über den Süßwert der einzelnen Zuckerart zu einer Bestimmung des Gesamtsüßwertes zu gelangen.

Es sei hier darauf hingewiesen, da $B$ neben der besonderen Eigenart des Geschmacks der einzelnen Säure- und Zuckerarten eine gegenseitige Beeinflussung des Geschmacks durch die Säuren und Zucker stattfindet, d.h. daß bei gleichem Säure- aber verschiedenem Zuckergehalt das zuckerreichere Gemisch weniger sauer schmeckt als das andere. Umgekehrt kann bei verschiedenem Säuregehalt und gleichem Zuckergehalt das säureärmere Gemisch süßer schmekken als das säurereichere.

Im Laufe des letzten Jahrzehntes wurden papierchromatographische Methoden zur Bestimmung der einzelnen Säure- und Zuckerarten entwickelt. Zusammenfassend haben CRAMER (I955), LEDERER (I955) und Linskens (I955) diese Methoden dargestellt. Wir haben versucht, aus der Fülle der vorhandenen Methoden diejenigen herauszufinden, die sich für die züchterischen Zwecke besonders gut eignen.

Zum Nachweis von Milchsäure, Bernsteinsäure, Apfelsäure, Zitronensäure, Weinsäure und Oxalsäure sowie von Fructose, Glucose, Saccharose, Maltose und Lactose hat sich folgende Methode bewährt:

Es werden Preßsäfte von Früchten und Gemüsen hergestellt. Vom Preßsaft wird mit der Mikropipette soviel auf den Staripunkt aufgetragen, daß ein Kreis von $4 \mathrm{~mm}$ Durchmesser gerade feucht wird. Das entspricht einer Lösungsmenge von etwa $0,002 \mathrm{ml}$ und bei einer $I \%$ igen Lösung einer Stoffmenge von etwa $20 \gamma$.

Die Chromatogramme laufen aufsteigend eindimensional.

Als Papier wird verwendet: Schleicher \& Schüll 2043 b Mgl.

Als Lösungsmittel hat sich Butanol-Eisessig-Wasser im Verhältnis 8:1:5 bewährt.

Die Laufzeit soll mindestens I 2 Stunden, zur klaren Trennung von Fructose und Glucose mindestens 24 Stunden betragen.

Die Temperatur während des Laufens muß konstant sein und sollte $25^{\circ}$ bis $26^{\circ} \mathrm{C}$ betragen. Niedrigere Temperaturen erfordern längere Laufzeiten.

Nach dem Herausnehmen aus dem Lösungsmittel wird das Chromatogramm bei etwa $20^{\circ} \mathrm{C}$ getrocknet.

Für die Säurebestimmung werden die Papiere nach der Trocknung bei etwa $20^{\circ} \mathrm{C}$ in säurefreiem Raum aufgehängt. Man besprüht sie mit einer Lösung von Bromphenolblau (nach Linskens: $40 \mathrm{mg}$ Indikator werden in $95 \mathrm{ml}$ Äthanol und $5 \mathrm{ml}$ Wasser gelöst und mit o, I $n$ Natronlauge auf $p_{n} 5$ (purpurner Farbton) gebracht. Kleine Abweichungen vom $\mathrm{p}_{H}$-Wert erwiesen sich nicht als störend).

Nach dem Sprühen läßt man das Chromatogramm bei etwa $20^{\circ}$ in säurefreiem Raum trocknen. Die Säuren erscheinen als gelbe Flecken auf blauviolettem Grund (abweichend von Linskens: purpurner Grund). Vergleicht man die drei Säurearten Wein-, Apfel- und Zitronensäure papierchromatographisch bei gleicher Konzentration und steigenden Reihen, so findet man, daß der Schwellenwert der Sichtbarkeit bei der Weinsäure niedriger als bei der Apfel- und Zitronensäure liegt. Bei geringen Konzentrationen bilden die Säuren Flecke, bei denen die Rf-Werte bestimmt werden können und konstant sind.

Bei zunehmender Konzentration zeigen sich immer stärkere Schwanzbildungen und Erhöhungen der Laufgeschwindigkeit. Da die Ortsveränderung der Konzentration proportional ist, läßt sich mit ihrer Hilfe eine grobe quantitative Bestimmung durchführen. Verschiedene organische Säuren beeinflussen sich gegenseitig in ihrer Laufgeschwindigkeit, je nach ihrer Konzentration in stärkerem oder schwächerem Maße.

Wir haben daher auf Grund von Säuregemischen bestimmter Konzentrationsstufen auf empirischem Wege die Säurebilder hergestellt, an Hand derer wir die Auswertung der Ergebnisse vornehmen können (Abb. I-6).

In Anbetracht der verschieden hohen Laufgeschwindigkeiten der Säuren bei verschiedener Konzentration empfiehlt es sich, die Identifizierung der Säurearten durch Zusatz der reinen Säuren zu dem zu prüfenden Material vorzunehmen (Abb. 7). Soll geprüft werden, welche Säuren in einem Material enthalten sind, das zwei Säureflecke ergibt, setzt man nacheinander jeweils Apfelsäure, Zitronensäure und Weinsäure zu. Bei Gleichartigkeit der Säuren gibt es eine Verstärkung eines Fleckes, bei Ungleichartigkeit eine Vermehrung der Fleckenzahl. Auf diese Weise kann man unabhängig vom Rf-Wert eine Identifizierung der Säuren vornehmen.

Säurechromatogramme sind sowohl gegenüber Säuredämpfen im Raum als auch gegenüber mit Säuren 
oder Laugen behandelten Chromatogrammen außerordentlich empfindlich (Zuckerentwicklung).

Auf Zucker werden die getrockneten Papiere mit einem Reagens ähnlich dem von LINSKENs entwickelt:

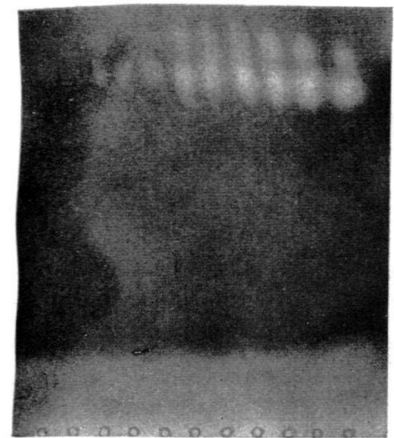

0
0
0
0

Abb. I. Milchsäure steigende Reihe $0,16-9,14^{\circ}$ (Steigerung um je $50 \%$ ).

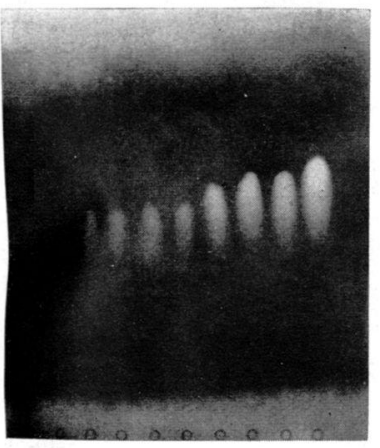

0
0
0
0

Abb. 3. Apfelsäurc steigende Reile $0,16-6,10 \%$ (Steigerung um je $50 \%$ ).

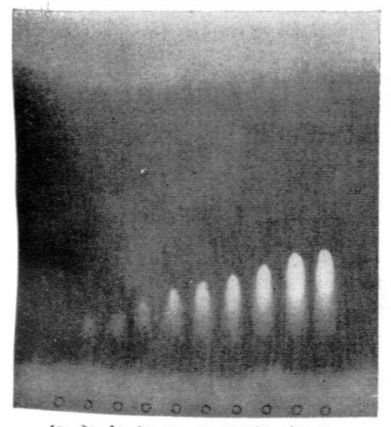

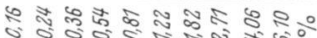

Abb. 5. Weinsäure steigende Reihe $0,16-6,0^{\circ}$ \% (Steigerung um je $50^{\circ} \%$ ).

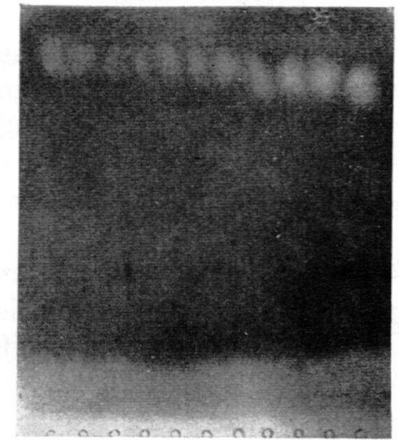

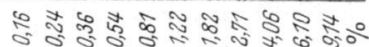

Abb. 2. Bernsteinsäure steigende Reihe $0,16-9,4^{n}$ (Steigerung um je $50 \%$ ).

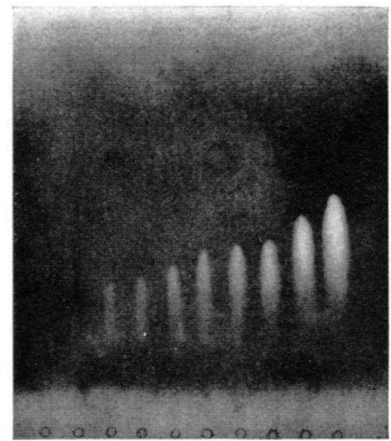

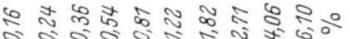

Abl). 4. Zitronensäure st ?igende Reihe $0,16-6,10^{\circ}$ (Stcigerung um je $50 \%$ ).

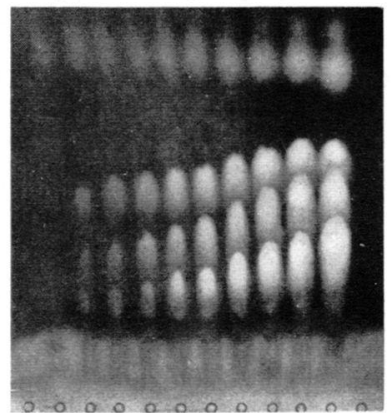

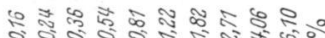

Abb. 6. Milch-, Bernstein-, ApfelZitronen- und Weinsäure.

$5 \mathrm{~g}$ Harnstoff löst man in $20 \mathrm{ml} 2 \mathrm{n}$ Salzsäure und $80 \mathrm{ml}$ Äthanol. Nach dem Besprühen wird an der Luft getrocknet und anschließend - abweichend von I.rsskens - genau 2 Minuten im Trockenschrank bei $\mathrm{I} 40^{\circ} \mathrm{C}$ in wasserdampfgesättigter Atmosphäre behandelt. Es muß dabei darauf hingewiesen werden, daß las Entwickeln der Säuren und Zucker möglichst in rerschiedenen Räumen vorgenommen werden sollte.

Der Rf-Wert der einzelnen Zuckerarten ist konstant und wird durch die Konzentration praktisch nicht beeinflußt :
Fructose:
Rf o,I8 blaugrau
Glucose:
Rf o,I3 rosabraun
Saccharose: Rf 0,08 blaugrau
Maltose:
Rf 0,05 blaugrau
Lactose:
Rf 0,04 blaugrau

Die Methode ist für Untersuchungen von Früchten und Gemüsen besonders gut geeignet, weil sie gleichzeitig die Hauptzuckerarten sichtbar macht und bei den relativ hohen Zuckerkonzentrationen der Preßsäfte gute Trennungen ermöglicht.

Auch die Identifizierung der Zucker kann im Zweifelsfalle unabhängig vom Rf-Wert wie bei den Säuren durch Zusatz reiner Zucker vorgenommen werden.

Man kann die Bestimmung der Säure und des Zuckers getrennt auf verschiedenen Blättern vornehmen. Für züchterische Zwecke empfiehlt es sich, aus Gründen der Arbeitsersparnis Säure und Zucker gleich zeitig auf einem Blatt $z u$ bestimmen. Man läßt das Chromatogramm in der beschriebenen Weise laufen und ermittelt nach dem Trocknen durch Bestimmung des Rf-Wertes die Lage des Fructosefleckes. Oberhalb dieses Fleckes wird dasChromatogrammzerschnitten und im Anschluß der obere Teil auf Säure und der untere auf Zucker entwickelt. Diese Methode ist nur anwendbar, wenn die in dem Untersuchungsmaterial nachzuweisenden Zucker- und Säurearten sich nicht auf dem Chromatogramm überschneiden.

Wir haben mit den reinen Zuckerarten Verdünnungsreihen angesetzt (Abb. 8, 9) und folgendes gefunden:

Bei gleichen Konzentrationsreihen haben auch die Zuckerarten

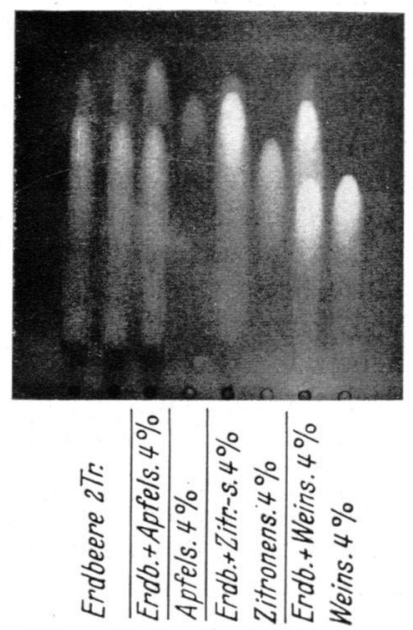

Alb. 7. Die Bestimmung der Säure arten von Erdbeeren durch Zusatz von jeweils Apfelsäure, Zitronensäure und Weinsaiure. Die Erdbecre enthält Apfel- und Zitronensäure.
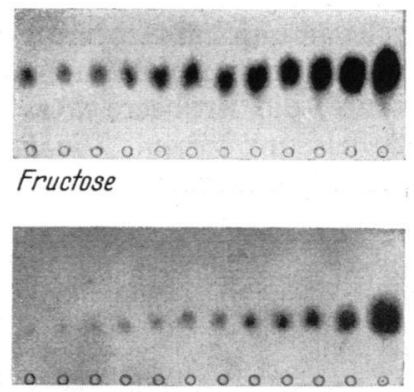

Glukose

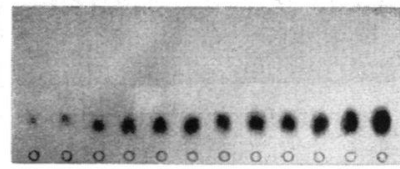

Saccharose

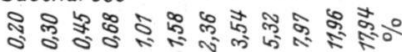

Abb. S. a) Fructose b) Glucose; c) Saccharose steigende Reihen der Zucker 0,20 bis ${ }_{17}, 94 \%$ (Steigerung um jc $50 \%$ ).

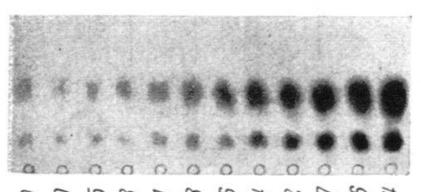

공 ₹

Abb. 9. Fructose, Glucose und Saccharose, steigende Reihe der drei Zucker $0,20-17,94 \%$ (Steigerung um je $50 \%$ ) verschiedene Schwellenwerte des Sichtbarwerdens: Glucose wird gegenüber Fructose und Saccharose erst bei höheren Konzentrationen sichtbar.

Mit Hilfe der beiden Methoden haben wir Sortimente von Obst-, Beerenobst- und Gemüsearten auf Säuren und Zucker untersucht. Es sollte dabei die 
Brauchbarkeit der Methoden und die Übereinstimmung der Chromatogramme mit dem Geschmack geprüft werden, das heißt, ob diese Methoden ausreichen, um die zwischen den Arten und innerhalb der Arten die zwischen den Sorten vorhandenen Unterschiede im Gehalt an verschiedenen Säure- und Zuckerarten qualitativ und quantitativ zu erfassen.

Es wurden folgende Obstarten untersucht: Zitrone, Pampelmuse, Apfelsine, Mandarine, Clementine, Banane, Weintraube, Ananas, Apfel, Birne, schwarze Johannisbeere, Himbeere, Erdbeere, Pflaume.

Auf Abb. Io ist das Ergebnis der papierchromatographischen Untersuchung auf Säure und Zucker von 5 Citrus-Arten wiedergegeben.

Es zeigt sich, da $\beta$ die Zitrone neben viel Zitronensäure Spuren an Apfelsäure, die Pampelmuse neben viel Zitronensäure kaum Apfelsäure, die Apfelsine neben viel Zitronensäure viel Apfelsäure, die Mandarine praktisch nur Zitronensäure und die Clementine wenig Zitronen- und relativ viel Apfelsäure enthält (Tab. Ia).

Die Zitrone hat im Gegensatz zu anderen CitrusFrüchten praktisch keine Saccharose und relativ wenig Fructose und Glucose, die Pampelmuse hat weniger Saccharose als Apfelsine, Mandarine und Clementine, die Mandarine die meiste Saccharose und die Clementine mehr Saccharose als die Apfelsine.

Abb. II zeigt, daß der Apfel nur Apfelsäure, die Birne Spuren von gleichfalls nur Apfelsäure, die schwarze Johannisbeere große Mengen Zitronensäure und Spuren Apfelsäure, die Himbeere nur Zitronensäure, die Erdbeere wenig Zitronensäure und wenig Apfelsäure haben. Die Pflaume besitzt neben recht viel Apfelsäure eine beträchtliche Menge Weinsäure.
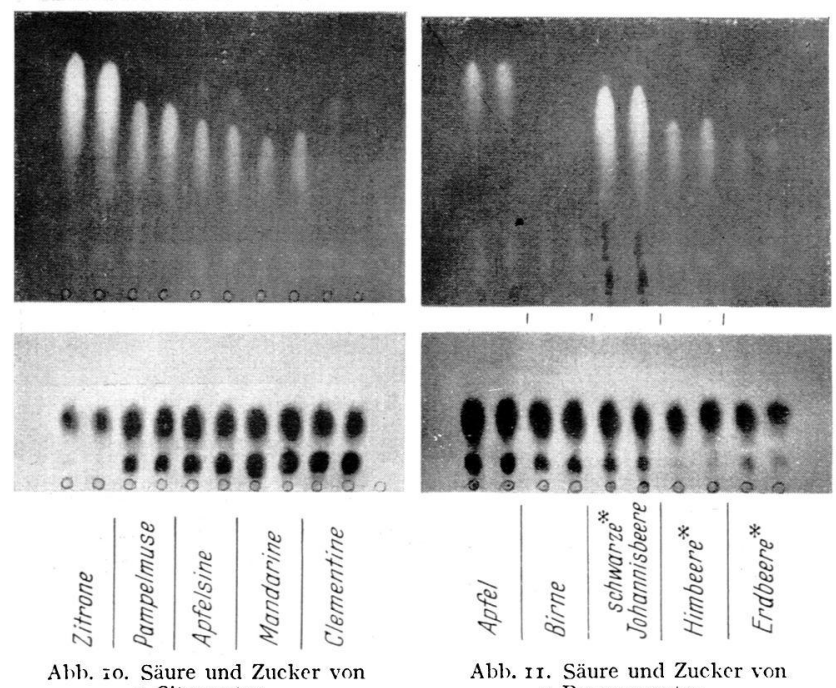

5 Citrusarten.

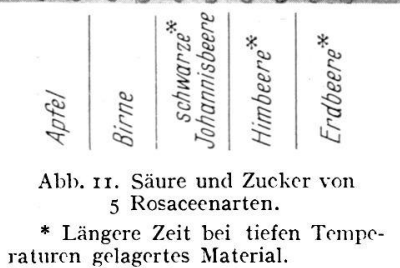

Tiefgefrorene Himbeeren und Erdbeeren enthalten keine bzw. wenig Saccharose, der Apfel (Cox Orange) viel Saccharose. Alle untersuchten Arten haben Fructose und Glucose. Die Pflaume nimmt auch hier, wie bei den Säuren, eine Sonderstellung ein, sie hat neben Fructose, Glucose und Saccharose noch eine weitere Zuckerart.

Die Ananas zeigt große Mengen Zitronensäure und etwas Apfelsäure, die Weintraube viel Apfelsäure, keine Zitronensäure und etwas Weinsäure.
Tabelle I a. Gegenïberstellung der papierchromatographisch gewonnenen Säure- und Zuckerergebnisse und der geschmacklichen Wahrnehmung bei Obstarten.

\section{Objekt \\ Zitrone} papierchromatographische Werte geschmacklich festgestellte
Menge an Gesamt-
-Säure -Zucker

Pampelmuse Apfelsine Mandarine Clementine Apfel Birne schw. Johannisbeere Himbeere* Endbeere* Ananas Banane Traube Pflaume* $\square$ 䀠
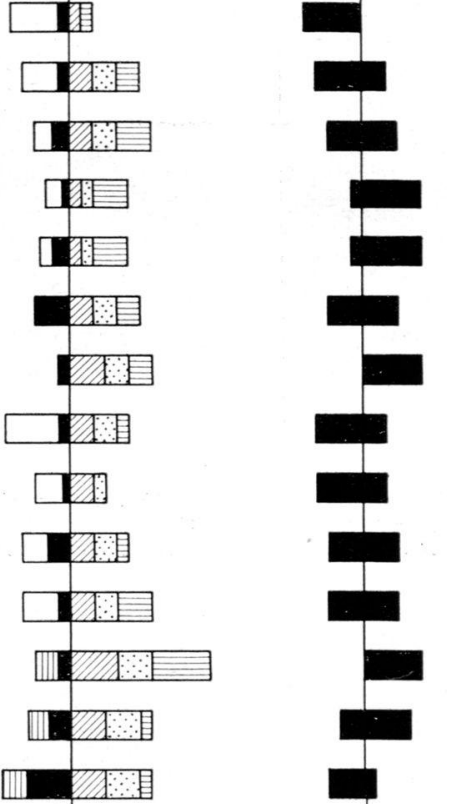
spezifischer
Geschmack IIIII

\section{Zeichenerklärung:}

\begin{tabular}{|c|c|c|}
\hline $\begin{array}{l}\text { Säuren } \\
\text { Apfe/säure } \\
\text { Z Zitronensäure } \\
\text { ⿴囗 Weinsäure }\end{array}$ & $\begin{array}{l}\text { Zucker } \\
\text { Fructose (Laevulose) } \\
\text { G Glucose } \\
\text { 首 Saccharose } \\
\text { Q Maltose } \\
\text { Lactose }\end{array}$ & $\begin{array}{l}\text { Aromastärke } \\
\text { 口 schwach } \\
\text { ⿴囗十 mittel } \\
\text { ⿴囗十 stark } \\
\text { - sehr stark }\end{array}$ \\
\hline
\end{tabular}

* Längere Zeit bei ticfen Tenperaturen gelagertes Material.

Tabelle I b. Gegenüberstellung der papierchromatographisch gewonnenen Säure- und Zuckerergebnisse und der geschmacklichen Wahrnohmung bei Apfelsinensorten.

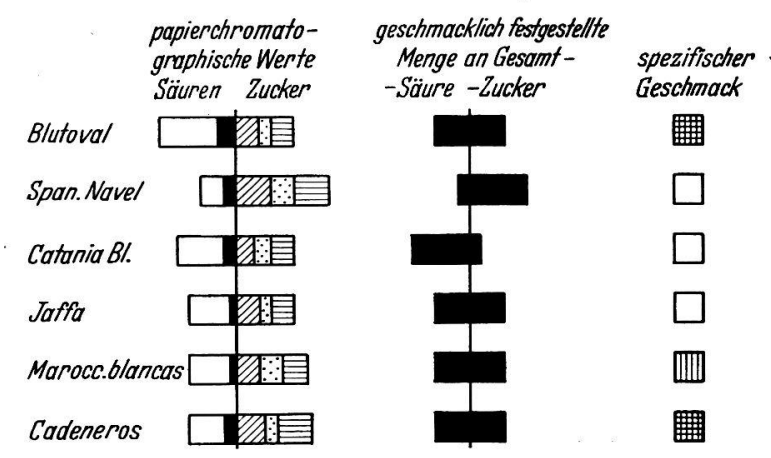

Während die Weintraube und die Tomate praktisch keine Saccharose enthalten, weist die Ananas viel Saccharose auf (Tab. Ia und I c).

Die Abb. I2 zeigt alle die Obst- und Gemüsearten, bei denen wir Weinsäure gefunden haben.

In Tab. I a haben wir die Ergebnisse der Papierchromatogramme dem vom Menschen festgestellten Geschmack gegenübergestellt. Auf Grund der Papierchromatogramme ist links von der Mittellinie der Gehalt an verschiedenen Säuren und rechts der Gehalt an verschiedenen Zuckern, nach Möglichkeit quantitativ eingetragen worden. Beim Geschmack ist links die Säurekomponente und rechts die Zuckerkompo- 
nente in ihrer Intensität veranschaulicht. Außerdem ist in der dritten Kolonne die Intensität des Aromas wiedergegeben.

Es zeigt sich, daß bis zu einem gewissen Grade eine Übereinstimmung zwischen dem Papierchromatogramm und dem geschmacklichen Test vorhanden ist.

Der saure Geschmack der Zitrone erklärt sich aus dem hohen Säuregehalt bei sehr geringem Zuckergehalt. Der angenehm süßsaure Geschmack von Pampelmuse und Apfelsine wird durch einen hohen Säureund ebenfalls hohen Zuckergehalt verursacht. Mandarinen schmecken deutlich süß, weil sie neben geringen Mengen von Zitronensäure einen hohen Saccharosegehalt haben, und bei den Clementinen läßt sich der besonders süße Geschmack auf den geringen Zitronen- und Apfelsäuregehalt bei großer Zuckermenge zurückführen.

Zusammenfassend können wir sagen, daß das Papierchromatogramm sowohl die qualitativen als auch die quantitativen Unterschiede bezüglich des Gehalts an einzelnen Säure- und Zuckerarten erkennen läßt.

Den Züchter interessiert aber weiterhin, ob auch den geschmacklichen Unterschieden von Sorte zu Sorte chemisch und papierchromatographisch faßbare Differenzen sowohl quantitativ als auch qualitativ zugrunde liegen.

Aus diesem Grunde haben wir Sorten ein- und derselben Art untersucht, und zwar von Apfelsinen, Äpfeln und Erdbeeren, letztere tiefgefroren.

Bei einer Reihe von Apfelsinensorten wurden deutliche Unterschiede im Säuregehalt festgestellt, während der Zuckergehalt aller Sorten etwa gleich war (Tab. Ib, Abb. r3).

Der kräftig säuerliche Geschmack der „Blut oval“ wird hauptsächlich durch das starke Vorhandensein

Tabelle I c. Gegenüberstellung der papierchromatographisch gewonnenen Säure- und Zuckerergebnisse und der geschmacklichen Wahrnehmung bei Gemüsearten.
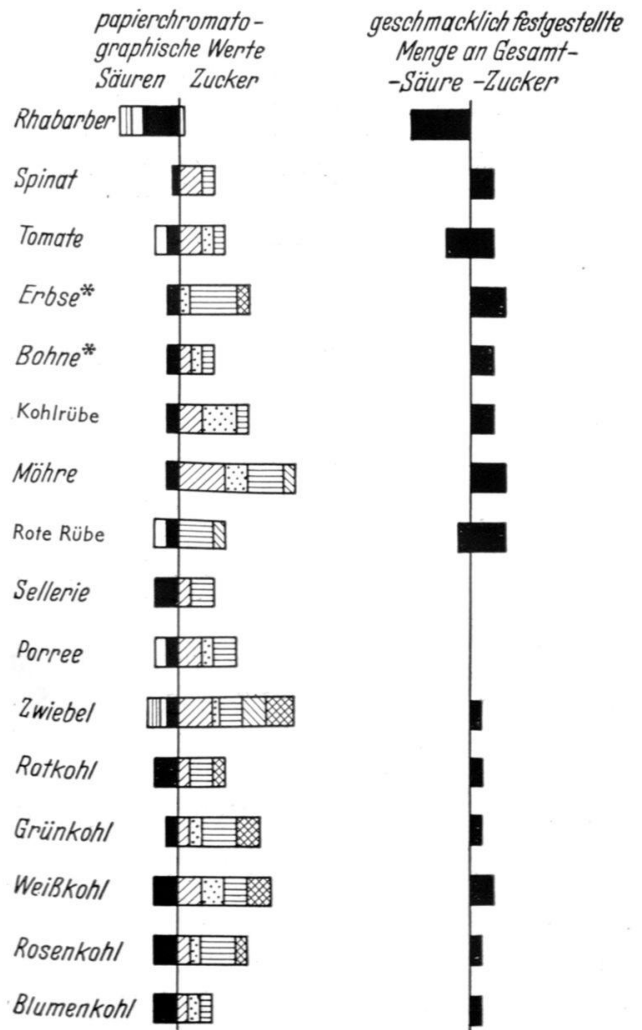

spezifischer Geschmack

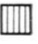

眏

von Zitronensäure neben einer beträchtlichen Menge an Apfelsäure hervorgerufen. Die spanische Navel hat neben wenig Zitronensäure und wenig Apfelsäure relativ mehr Zucker als die anderen Sorten (Fructose, Glucose, Saccharose) und schmeckt daher deutlich süß. Die anderen vier von uns untersuchten Sorten (Catania Blut, Jaffa, Maroccanische Blanca und Cade-
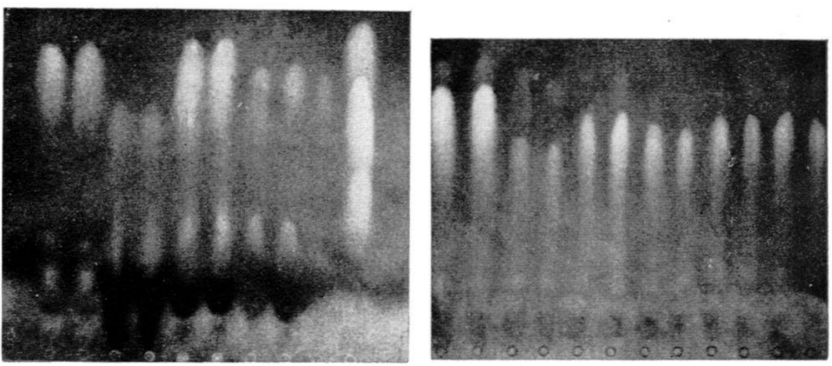

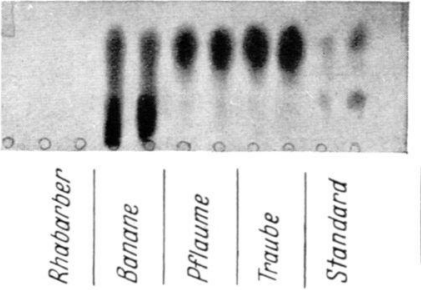

Abb. r2. Obst- und Gemüsearten, die nur Apfel- und Weinsäure, aber keine Zitronensäure enthalten.

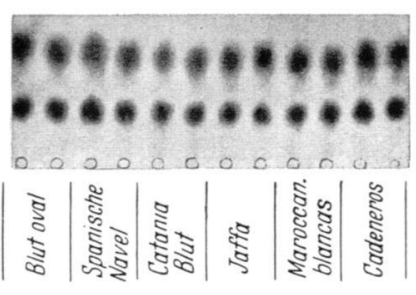
6 Apfelsinensorten.
Abb. r3. Säure und Zucker von

nero) schmecken zu gleichen Teilen sauer und süß, sie haben auch ungefähr gleiche Mengen an Fructose, Glucose und Saccharose.

Die papierchromatographische Untersuchung eines kleinen Apfelsortiments ergab deutliche quantitative Unterschiede im Säure- und im Zuckergehalt bei den einzelnen Sorten. An Säuren enthalten die Äpfel nur Apfelsäure, an Zuckern Fructose, Glucose und Saccharose.

Bei den Äpfeln sind der Winesape, der Herbstprinz und Schöner von Boskoop auf Grund des hohen Säuregehaltes besonders sauer, während Martini und Sternreinette wenig Apfelsäure, die Sternreinette außerdem besonders wenig Saccharose enthalten. Der saure Geschmack der Sternreinette dürfte auf den geringen Zuckergehalt zurückzuführen sein.

Die Geschmacksunterschiede bei den Apfelsorten beruhen also auch auf dem unterschiedlichen Gehalt an Säure, in diesem Fall Apfelsäure, und den verschiedenen Zuckern (Abb. I4).

Es zeigt sich, daß bereits in einem kleinen Erdbeersortiment von 5 Sorten eine Sorte (SENGA I46) mit besonders hohem Zitronensäuregehalt und eine zweite Sorte (Georg Soltwedel) mit besonders niedrigem Apfelsäuregehalt vorhanden ist.

Auch beim Zucker gibt es erhebliche Unterschiede. Die Sorten SENGA-SENGANA und SENGA 29 enthalten nach Lagerung bei tiefen Temperaturen viel, die übrigen praktisch keinen Rohrzucker.

Auch bei den Erdbeeren zeigt sich eine Parallele zwischen der geschmacklichen Feststellung und dem Chromatogramm. Dabei spielen die einzelnen Komponenten eine sehr unterschiedliche Rolle. Der Mensch ist nicht in der Lage auszusagen, ob eine Frucht süß schmeckt, weil sie viel Zucker oder aber wenig Säure enthält. Bei Geschmacksproben an einer großen Zahl von tiefgefrorenen Erdbeerklonen wurde im Vergleich 
mit den Chromatogrammen festgestellt, daß Unterschiede in Gehalt und Menge der verschiedenen Zuckerarten nur ziemlich schwer wahrgenommen werden. Quantitative Differenzen imSäuregehalt lassen sich dagegen vom Menschen recht gut bestimmen (Abb. I5).
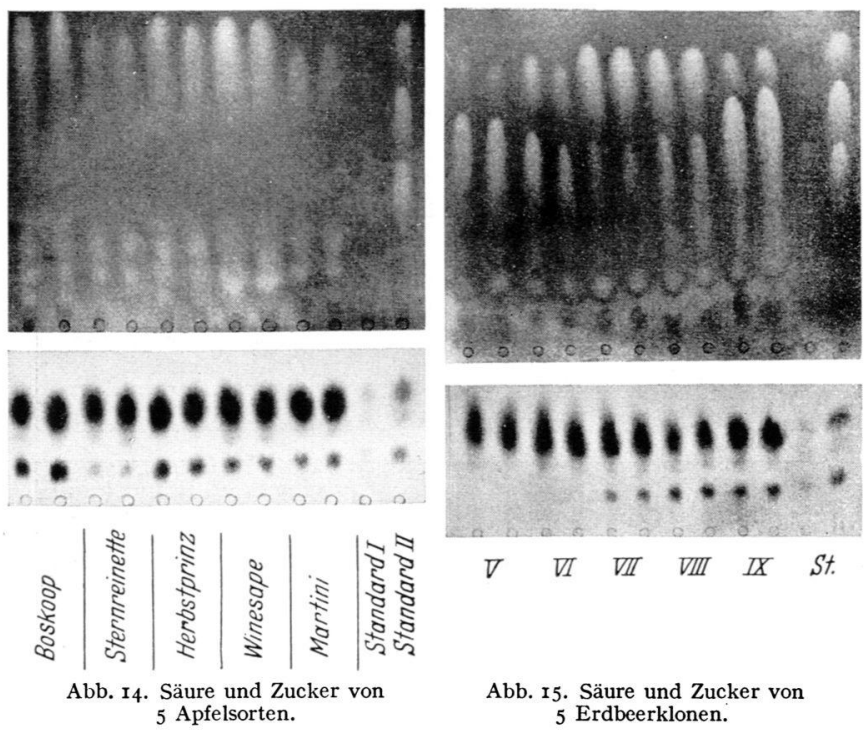

Als frisch und angenehm sauer wird die Zitronensäure, als unangenehm sauer die Apfelsäure empfunden. Ein gewisser Gehalt an Zitronensäure und ein sehr geringes Vorkommen von Apfelsäure können daher zum gleichen Ergebnis, einem angenehmen
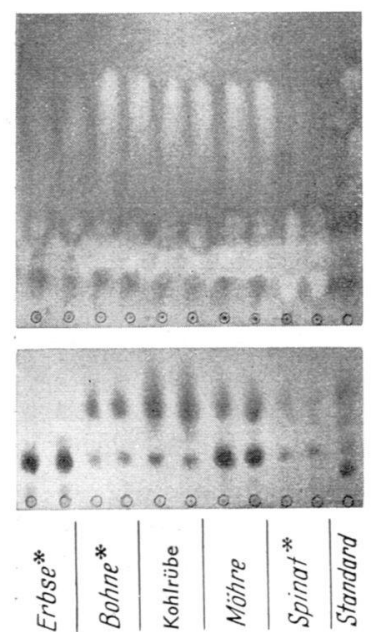

Abb. I6. Säure und Zucker von 5 Gemüsearten.

* Längere Zeit bei tiefen Temperaturen gelagertes Material.
Geschmack, führen. Das zeigt sich bei den Sorten SENGA I 46 und Georg Soltwedel. Die übrigen Sorten schmecken trotz hohen Zuckergehaltes säuerlich, weil sie daneben noch viel Apfel- und Zitronensäure enthalten.

Sowohl bei den Apfelsinensorten als auch bei den Äpfeln und Erdbeeren ergeben sich bis zu einem gewissen Grade Übereinstimmungen zwischen der papierchromatographischen Feststellung der Säuren und Zucker mit dem Geschmack. Im Rahmen unserer papierchromatographischen Untersuchungen wurden folgende Gemüsearten geprüft:

Erbse, Bohne, Kohlrübe, Möhre, Spinat, Rhabarber, Tomate, Rote Rübe, Sellerie, Porree, Rotkohl, Weißkohl, Grünkohl, Rosenkohl und Blumenkohl (Abb. I6, I7, I8, Tab. Ic).

Erbse, Bohne, Kohlrübe, Möhre und die Kohlarten enthalten nur Apfelsäure; Rhabarber, Tomate, Rote Rübe und Porree daneben etwas Zitronensäure; Rhabarber außerdem noch Weinsäure.

Der Zuckergehalt der Gemüse ist durchweg überraschend hoch.

Außer Erbse, Rote Rübe und Rhabarber haben alle Gemüse Fructose und Glucose. Saccharose ist in allen Arten außer bei Tomate und Rhabarber vorhanden.
Erbse, Möhre und Rote Rübe zeichnen sich durch besonders hohen Saccharosegehalt aus.

Erbse, Möhre, Rote Rübe, Porree und die Kohlarten besitzen außerdem noch Maltose bzw. Lactose.

Wir haben innerhalb einer Sorte von Roten Rüben über 400 über Winter gelagerte Individuen untersucht, um festzustellen, wiegroß die Variationsbreite bezüglich des Zuckergehaltes ist.

Die Rüben enthalten in der Regel große Mengen Saccharose und praktisch keine Fructose und Glucose.

Wir fanden ein einziges Individuum, das einen hohen Gehalt an Fructose und Glucose aufwies, dagegen nur wenig Saccharose (Abb. I9). Bei der refraktometrischen Untersuchung hatte sich diese Rübe von normalen Rüben im Gehalt an im Zellsaft gelösten Stoffen praktisch nicht unterschieden. Die Auffindung einer solchen bezüglich der Zuckerzusammensetzung anormalen Rübe wäre mit Hilfe des Refraktometers nicht möglich gewesen.

Dieses Beispiel weist darauf hin, daß die Ausbildung derSaccharosein Rüben und damit wohl auch in Zuckerrüben nicht absolut artbedingt ist.

Alle Gemüse, die nur wenig Apfelsäure besitzen, schmecken nicht sauer, während die Arten, die außerdem noch Zitronensäure zeigen, einen leichten Säure-
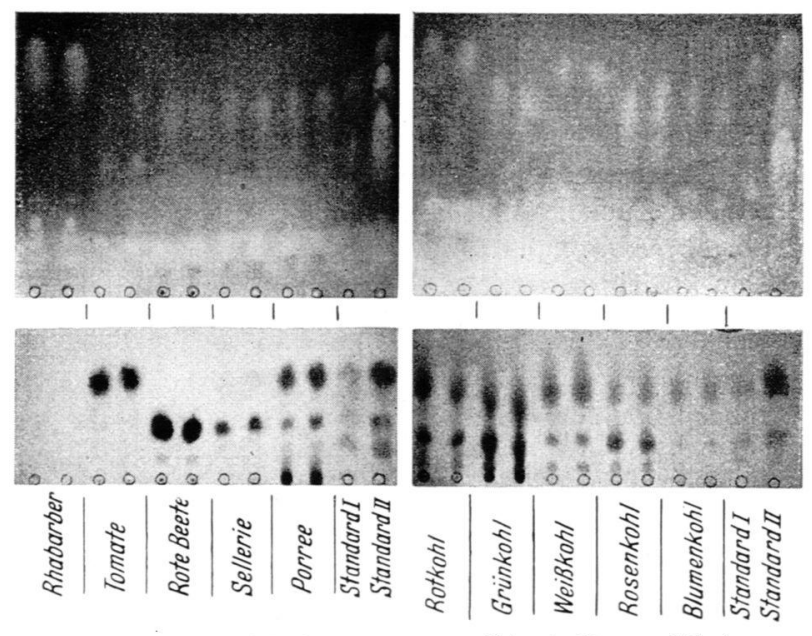

Abb. I7. Säure und Zucker von 5 Gemüsearten.

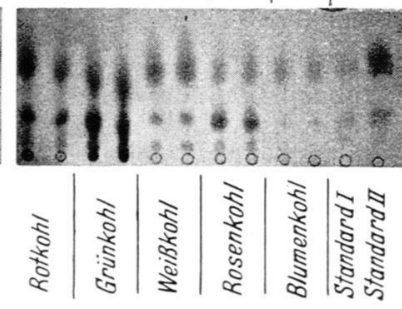

Abb. 18. Säure und Zucker von 5 Kohlarten.

Abb. 19. Der Zuckergehalt verschiedener Individuen von Roten Rüben. Nr, 357: die einzige vonetwa 400 Rüben, die praktisch keine Saccharose, dagegen viel Fructose und Glucose enthält.

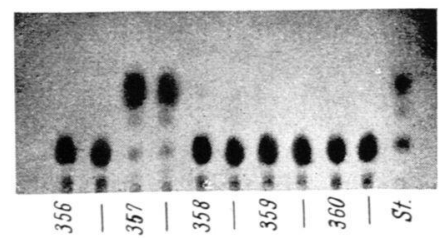

geschmack haben, z. B. die Tomate. Der Rhabarber mit sehr viel Apfelsäure, etwas Zitronen- und Weinsäure schmeckt sehr stark sauer, zumal er keinen Zucker besitzt.

Von dem in den Gemüsearten chromatographisch festgestellten Zucker ist bei einigen geschmacklich nicht viel wahrzunehmen. Das mag seinen Grund in dem sehr stark ausgeprägten ,Aroma“ dieser Gemüsearten haben z. B. bei Kohl, Kohlrüben und $Z$ wiebeln.

Die im Papierchromatogramm sichtbar gemachte Menge an Säuren tritt geschmacklich nicht in Erscheinung. Eine wäßrige Lösung von Apfelsäure in gleicher Konzentration dagegen schmeckt deutlich sauer, d.h. daß die Gemüse Stoffe zu enthalten scheinen, 
die den Säuregeschmack dämpfen. $\mathrm{Ob}$ es sich hierbei nur um Zucker oder auch Salze oder andere Stoffe handelt, wird noch $\mathrm{zu}$ klären sein. Wir finden auf den Papierchromatogrammen der Gemüse neben den gelben Säureflecken mehr oder weniger ausgeprägte blaue Flecken, die auf die Anwesenheit von Stoffen mit alkalischer Reaktion hindeuten.

Diese blauen Flecken können gegenüber den gelben Flecken mit Hilfe eines Diapositiv-Filmes gut sichtbar gemacht werden (Abb. 20). Es wäre für den Züchter von allergrößtem Interesse $z u$ erfahren, welche Rolle die alkalischen Komponenten in geschmacklicher Beziehung spielen. Bei Klärung dieser Frage wäre er in der Lage, nicht nur den Säure- und Zuckerkomplex, sondern auch quantitativ den alkalischen Komplex mitzubestimmen und planmäßige Veränderungen an diesem Komplex vorzunehmen.

Der Züchter von

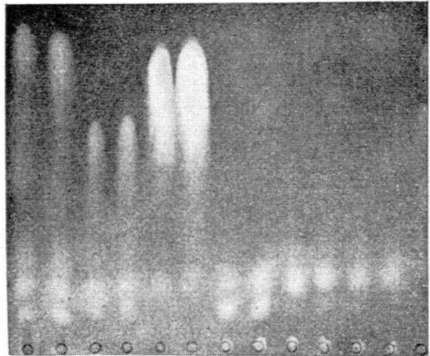

Agfa AGEPE

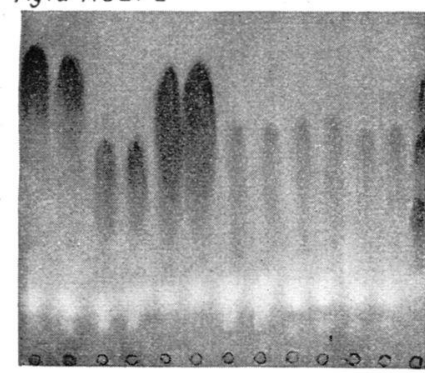

Perutz Diapositivfilm

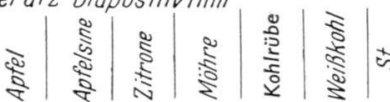

Abb. 20. Reproduktion der Säuren. a) mit Negativ-Film (Agfa AGEPE); b) mit Positiv-Film (Perutz-Diapositiv).

a) Säuren und Basen erscheinen hell;

b) Säuren erscheinen dunkel, Basen hell.

rungen ein. Es mußte geklärt werden, ob entsprechende chemische Veränderungen durch das Tiefgefrieren und Wiederauftauen nachzuweisen sind. Wir haben frische und im Vergleich dazu tiefgefrorene und wiederaufgetaute Beerenobstarten papierchromatographisch untersucht und dabei festgestellt, daß bei nichtblanchierten Früchten nach längerer Lagerzeit die Sacharose verschwindet. Wir konnten noch nicht klären, ob mit dem Verschwinden des Saccharosegehaltes eine Steigerung des Fructose- und Glucosegehaltes parallel geht. Der Züchter muß also, wenn er durch Tiefgefrieren konserviertes, unblanchiertes Zuchtmaterial untersuchen will, mit der Veränderung des Saccharosegehaltes rechnen.

Um den optimalen Zeitpunkt für die Untersuchung, bzw. für die Einlagerung von Früchten festzustellen, haben wir den Vorgang der Reife papierchromatographisch verfolgt. Da wir unsere Untersuchungen im Laufe des Winters vorgenommen haben, standen uns für diesen Zweck praktisch nur Bananen zur Verfü- gung. Sie wurden im unreifen und reifen Zustand untersucht (Abb. 2I).

Es zeigte sich während des Reifeprozesses eine $\mathrm{Zu}$ nahme des Säuregehaltes und eine sehr starke $\mathbf{Z u}$ nahme des Fructose- und Saccharosegehaltes. Die $\mathrm{Zu}$ nahme des Säuregehaltes war geschmacklich wegen
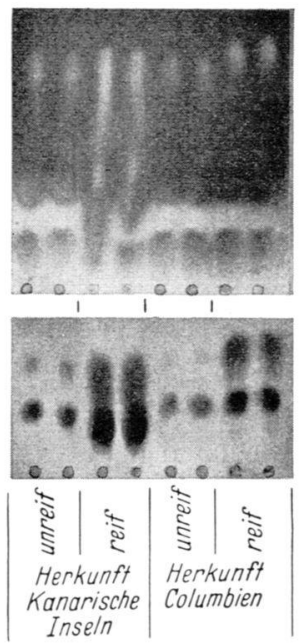

Abb. 2r. Die Veränderung des Säureund Zuckergehaltes von $z$ wei Bananensorten während der Reife.
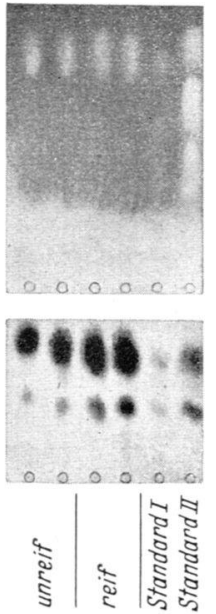

Abb. 22. Die Veränderung des Säureund Zuckergehaltes der Apfelsorte Ontario während der Reife. des stark gesteigerten Zuckergehaltes nicht wahrnehmbar. Auch bei Äpfeln stieg zunächst der Säuregehalt im Zusammenhang mit dem Reifen. Es war eine starke Zuckerzunahme festzustellen, die wiederum die Zunahme des Säuregehaltes geschmacklich nicht in Erscheinung treten ließ (Abb. 22).

Wir haben untersucht, welche Beziehungen zwischen der Ausprägung des Zucker- und Säuregehaltes und der Lichteinwirkung bestehen.
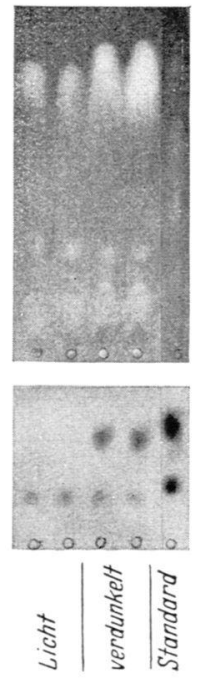

a

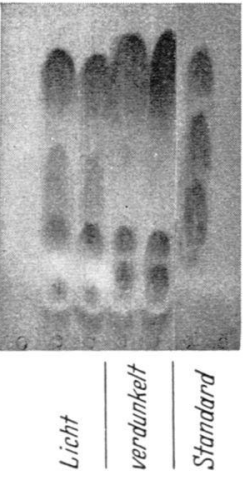

b
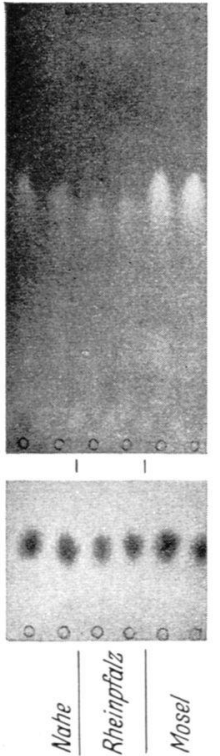

Abb. 24. Der Säureund Zuckergehalt von 3 deutschen

Die Unterschiede im Säure- und Zuckergehalt bzw. im Säuregehalt bei unter
Licht und unter LichtausschluB kultiviertem a) mit Negativirimer.

b) mit Positiv-Film (Perutz-Diapositiv)

Wir haben zunächst verdunkelte und nicht verdunkelte Rhabarberpflanzen geprüft. Es wurde festgestellt, daß die Verdunkelung die Ausbildung der Apfelsäure stark fördert und die Ausbildung der Zitronensäure verhindert (Abb. 23a und b). 
Der unverdunkelte Rhabarber enthält praktisch keine Fructose, der verdunkelte hat einen sehr hohen Fructosegehalt.

Die Saccharose bleibt bei unverdunkeltem und verdunkeltem Rhabarber auf gleicher Höhe.

Für den Rhabarber hat diese Feststellung unmittelbar praktische Bedeutung, weil man Rhabarber im Frühjahr in verdunkelten Warmkästen treibt.

Auch Spinat wird im Frühjahr im Gewächshaus unter wesentlich ungünstigeren Belichtungsverhältnissen als im Freiland angezogen.

Wir haben im AnschluB an die Untersuchungen der Obstarten und der Gemüse auch Obsterzeugnisse geprüft. Beim Wein können die Veränderungen der primär vorhandenen Stoffe (Säuren und Zucker), die im Zuge der Gärung entstandenen, z. B. Bernsteinsäure) und die sekundär zugesetzten Stoffe (Saccharose, Zitronensäure) papierchromatographisch sichtbar gemacht werden. Vor allen Dingen aber werden auch die Unterschiede im Säure- und Zuckergehalt bei den einzelnen Weinsorten sichtbar, z. B. höherer Apfelsäuregehalt beim Mosel gegenüber dem beim Rheinwein (Abb. 24).

Die bisher gewonnenen Ergebnisse kann man so deuten, daß die Papierchromatographie dem Züchter die Möglichkeit gibt, eine chemische Vorauslese auf Geschmack durchzuführen. Sie erfüllt die Anforderungen, die man an eine Schnellbestimmungsmethode stellt. Der Züchter ist somit in der Lage, mit Hilfe papierchromatographischer Methoden auch ein zahlenmäßig großes Material zu bewältigen.

Die Untersuchungen haben gezeigt, daß sowohl die Arten als auch innerhalb der Arten die Sorten und auch die Individuen sehr erhebliche papierchromatographisch nachweisbare Unterschiede bezüglich des Gehaltes an einzelnen Zucker- und Säurearten aufweisen.

Es ist vielfach geäußert worden, daß die Papierchromatographie wohl den qualitativen Nachweis chemischer Stoffe ermöglicht, daß sie aber die Voraussetzungen zur quantitativen Erfassung der Stoffe nicht besitzt.

Unsere Ergebnisse zeigen, daß die Unterschiede von Art $\mathrm{zu}$ Art, von Sorte $\mathrm{zu}$ Sorte und von Individuum zu Individuum jeweils so gro $B$ sind, $\mathrm{da} B$ die papierchromatographische Erfassung der vorhandenen Unterschiede durchaus ausreicht und auch die Möglichkeit bietet, quantitativ zu arbeiten.

Es ist zu vermuten, daß für jede einzelne Zuckerund Säureart autonome Gene verantwortlich sind, d.h. daß die einzelnen Zucker- und Säurearten nicht wie die verschiedenen Alkaloide der Lupine durch ein Gen in ihrer Ausbildung beeinflußt werden. Das bedeutet züchterisch, daß wir in der Lage sind, eine beliebige Synthese des Zucker- und Säurekomplexes durchzuführen.

Wir können bei den Erdbeeren Formen mit besonders niedrigem Apfelsäuregehalt und hohem Zitronensäuregehalt mit Formen kreuzen, die einen hohen Apfelsäuregehalt und niedrigen Zitronensäuregehalt aufweisen, um Formen zu züchten, die entweder gleichzeitig arm an Apfel- und Zitronensäure oder gleichzeitig reich an beiden Säuren sind. Dasselbe gilt auch bezüglich der Zuckerarten.

Vom physiologischen Standpunkt aus sind wir in der Lage, den ReifeprozeB bis in alle Einzelheiten zu verfolgen.

Von züchterischen Problemen, die nicht unmittelbar mit dem Geschmack zusammenhängen, können z.B. folgende gelöst werden:

Die Zuckerrübe soll, da aus ihr Saccharose gewonnen wird, ausschließlich Saccharose enthalten. Die Auslese auf hohen Zuckergehalt wird von den meisten Züchtern mit Hilfe des Refraktometers durchgeführt (früher wurde die Auslese mit Hilfe des Polarimeters vorgenommen, heute verwendet man das Polarimeter in kleinem Umfang nur als Ergänzung zur refraktometrischen Untersuchung).

Mit Hilfe des Refraktometers wird die Summe der im Zellsaft gelösten Stoffe bestimmt (Summe der Brechungsindices). Es besteht die Möglichkeit, daß bei der Auslese mit Hilfe des Refraktometers Rüben ausgelesen werden, die neben Saccharose auch andere Zuckerarten oder sogar nur andere Zuckerarten enthalten, wie wir an Roten Rüben nachweisen konnten.

Es würde sich empfehlen, in der Zuckerrübenzüchtung mit Hilfe der Papierchromatographie eine laufende Kontrolle des Zuchtmaterials bezüglich der Zusammensetzung des Zuckers durchzuführen und nur solche Individuen zur züchterischen Weiterbearbeitung und Vermehrung hinzuzuziehen, die ausschließlich Saccharose ausbilden. Es ergibt sich aber auch eine weitere züchterische Möglichkeit, nämlich Zuckerrüben und auch Gemüserüben (Rote Rüben) zu züchten, die keine Saccharose, sondern nur Fructose enthalten. Einmal wäre hierdurch die Möglichkeit gegeben, Fructose in größerem Umfang herzustellen und zweitens ein Gemüse zu erzeugen, das ausschließlich Fructose enthält. Die Erzeugung von nur fructosehaltigen Nahrungsmitteln wäre für die Ernährung von Diabetikern von Bedeutung.

Mit Hilfe der Papierchromatographie ist der Züchter erstmalig in der Lage, die Grundlagen des Geschmacks, soweit es den Komplex Säure und den Komplex Zucker betrifft, qualitativ und quantitativ zu erfassen. Er führt eine Analyse der Teileigenschaften durch und kann, wenn sich die Teileigenschaften autonom vererben, die gewünschten Teileigenschaften beliebig kombinieren. Mit Hilfe derartiger planmäßiger Kombinationen kann ein bestimmter gewünschter Geschmack synthetisiert werden. Es dürfte nur eine Frage der Zeit sein, daß man auch die Salze und Basen und einen Teil der Aromastoffe papierchromatographisch erfassen kann, so daß man in der Lage sein wird, weitere Teilkomplexe des Geschmacks zu analysieren und anschließend $\mathrm{zu}$ synthetisieren.

$$
\text { Literatur }
$$

I. Cramer, Friedrich: Papierchromatographie. 3. ergänzte Auflage. Verlag Chemie G.m.b.H., Weinheim/ Bergstraße r954. - 2. Linskens, H. F.: Papierchromatographie in der Botanik. Springer-Verlag, Berlin-Göttingen-Heidelberg I955. - 3. Moncrieff, R. W.: The Chemical Senses. Leonhard Hill Ltd., London 195x. 4. Lederer, E. \& M. : Chromatographie. Elsevier Comp., London 1955. 